\title{
Tetrandrine Suppresses Cancer Angiogenesis and Metastasis in 4T1 Tumor Bearing Mice
}

\author{
Jian-Li Gao, ${ }^{1}$ Xing Ji, ${ }^{1}$ Tong-Chuan He, ${ }^{2}$ Qi Zhang, ${ }^{3}$ Kai He, ${ }^{4}$ Yan Zhao, ${ }^{1}$ \\ Su-Hong Chen, ${ }^{3}$ and Gui-Yuan $\mathrm{Lv}^{1}$ \\ ${ }^{1}$ Zhejiang Chinese Medical University, Hangzhou, Zhejiang 310053, China \\ ${ }^{2}$ Molecular Oncology Laboratory, Department of Surgery, The University of Chicago Medical Center, Chicago, \\ IL 60637, USA \\ ${ }^{3}$ Wenzhou Medical College, Wenzhou, Zhejiang 325035, China \\ ${ }^{4}$ The First Affiliated Hospital of Zhejiang University, Hangzhou, Zhejiang 310009, China \\ Correspondence should be addressed to Jian-Li Gao; jianligao@gmail.com and Gui-Yuan Lv; lv.gy@263.net
}

Received 29 March 2013; Accepted 26 April 2013

Academic Editor: Thomas Efferth

Copyright (C) 2013 Jian-Li Gao et al. This is an open access article distributed under the Creative Commons Attribution License, which permits unrestricted use, distribution, and reproduction in any medium, provided the original work is properly cited.

\begin{abstract}
Metastasis remains the most deadly aspect of cancer and still evades direct treatment. Thus, there is a great need to develop new treatment regimens to suppress tumor cells that have escaped surgical removal or that may have already disseminated. We have found that tetrandrine (TET) exhibits anticolon cancer activity. Here, we investigate the inhibition effect of TET to breast cancer metastasis, angiogenesis and its molecular basis underlying TET's anticancer activity. We compare TET with chemotherapy drug doxorubicin in $4 \mathrm{~T} 1$ tumor bearing BALB/c mice model and find that TET exhibits an anticancer metastatic and antiangiogenic activities better than those of doxorubicin. The lung metastatic sites were decreased by TET, which is confirmed by bioluminescence imaging in vivo. On the other hand, laser doppler perfusion imaging (LDI) was used for measuring the blood flow of tumor in 4T1tumor bearing mice. As a result, the local blood perfusion of tumor was markedly decreased by TET after 3 weeks. Mechanistically, TET treatment leads to a decrease in p-ERK level and an increase in NF- $\kappa$ B levels in HUVECs. TET also regulated metastatic and angiogenic related proteins, including vascular endothelial growth factor, hypoxia-inducible factor-1 $\alpha$, integrin $\beta 5$, endothelial cell specific molecule-1, and intercellular adhesion molecule-1 in vivo.
\end{abstract}

\section{Introduction}

Tetrandrine (TET), a bisbenzylisoquinoline alkaloid isolated from the dried root of Stephania tetrandra (or hang fang ji) of the Menispermaceae, is a bioactive alkaloid with a molecular weight of $622.76 \mathrm{~g} / \mathrm{mol}$. Many reports indicated that TET exhibits very broad pharmacological actions, including immunomodulating, antihepatofibrogenetic, antiinflammatory, antiarrhythmic, antiportal hypertension, anticancer, and neuroprotective activities [1]. The beneficial effects of TET on tumor cell cytotoxicity and radiosensitization, multidrug resistance, normal tissue radioprotection, and angiogenesis are most promising and deserve great attention $[2,3]$. Several investigations indicated that TET generally presents its anticancer effects in the micromolar concentrations on clone, leukemia, bladder, hepatoma, and lung cancer in vitro [4-8].

In our previous research, we have found that TET exhibits significant anticancer activity in colon cancer line HCT116. Mechanistically, the inhibitory effect of TET on colon cancer cells may be at least in part mediated by targeting $\beta$-catenin activity, and the sensitivity of cancer cells to TET may be determined by the functional status of $\beta$-catenin [9].

Nevertheless, with the development of clinical surgical treatment, the growth of primary tumor was no longer the critical element influencing the overall survival in cancer patients. Prevention of metastasis and more effective treatment of cancer metastasis are necessary for cancer therapy. Thus, antimetastasis therapy that targets tumor cells escaped 
surgical removal or already disseminated, and tumor angiogenic process has a better chance of success. Although several rational lines of evidence support the application of TET as an anticancer metastatic agent [10], the cellular mechanisms underlying the antiangiogenic and antimetastatic effects of TET activation in tumor cells remain elusive.

In this study, we use a mouse model for stage IV breast cancer (4T1 tumor bearing BALB/c mice model) for evaluating the antimetastatic effect of TET in lower concentration $(10 \mathrm{mg} / \mathrm{kg} / \mathrm{d}$, which could not change the growth of tumor mass). Meanwhile, laser Doppler imager (LDI) was used for measuring blood perfusion of the tumor bearing area, which could measure the local blood flow and vascular network and reflect the angiogenic activity of the tumor bearing mice indirectly. Our results show that TET could significantly inhibit endothelial cell (EC) proliferation, adhesion, migration, invasion, and tube formation by targeting angiogenic factors, namely vascular endothelial growth factor (VEGF) and hypoxia-Inducible factor- $1 \alpha$ (HIF- $1 \alpha)$, as well as adhesion factors, such as integrin $\beta 5$, endothelial cell specific molecule1 (ESM-1), and intercellular adhesion molecule-1 (ICAM-1), and by interfering with the ERK pathway, leading to the suppression of tumor metastasis and tumor angiogenesis.

\section{Materials and Methods}

2.1. Cell Culture and Animals. Mouse breast cancer 4T1 and HEK-293 cells were purchased from American Type Culture Collection (ATCC, Manassas, VA, USA) and grown in the DMEM (Invitrogen, Carlsbad, CA, USA) supplemented with 10\% FBS (Hyclone, Logan, UT, USA) and $50 \mathrm{U}$ penicillin/streptomycin. Human umbilical vein endothelial cell line (HUVEC) was immortalized as described in [11] and maintained in RPMI 1640 medium (Invitrogen, Carlsbad, CA, USA) supplemented with 15\% FBS, 2 mM L-glutamate, $100 \mathrm{U} / \mathrm{mL}$ penicillin and $100 \mu \mathrm{g} / \mathrm{mL}$ streptomycin. All cells were cultured at $37^{\circ} \mathrm{C}$ in a $5 \% \mathrm{CO}_{2}$ incubator.

Female BALB/c mice (4-5 weeks old) were purchased from Shanghai Lab. Animal Research Center and maintained at the animal facility of Experimental Animal Research Center of Zhejiang Chinese Medical University. All procedures were performed according to protocols following the guidelines for the Use and Care of Laboratory Animals published by the US National Institutes of Health $(\mathrm{NIH}$ Publication no. 85-23, revised 1996).

2.2. Chemicals and Drug Preparations. TET and doxorubicin (Dox) were purchased from Sigma-Aldrich (St. Louis, MO, USA). These compounds were dissolved in DMSO to make stock solutions and were kept at $-20^{\circ} \mathrm{C}$ as aliquots. The solution was diluted with Milli-Q water into $2 \mathrm{mg} / \mathrm{mL}$ and ultrasonificated into a fine suspension before in vivo use. $0.1 \%$ DMSO was used in vehicle group in in vitro assays.

2.3. Establishment of Stably Tagged 4T1-Luc Cell Lines. The 4T1 cells were stably transduced with firefly luciferase by using a retroviral vector expressing firefly luciferase as described in [12]. Briefly, recombinant retrovirus was packaged in HEK-293 cells by cotransfecting cells with pSEBLuc and pAmpho packaging plasmid using LipofectAMINE (Invitrogen). Pooled stable cells were selected with blasticidin $\mathrm{S}(6 \mu \mathrm{g} / \mathrm{mL})$ for 7 days. The firefly luciferase activity was confirmed by using Promega's Luciferase Assay kit (Promega, Madison, WI, USA).

2.4. MTT Proliferation Assay. A modified MTT assay was used to examine the cell proliferation as described in [13]. Briefly, iHUVEC $\left(1 \times 10^{4}\right.$ cells/well, 50-70\% density) cells were seeded in 96-well plates. Drugs were added to the cells at variable concentrations or solvent control (0.1\% DMSO). At $48 \mathrm{~h}$ after treatment, $15 \mu \mathrm{L}$ MTT dye solution was added to each well and incubated for additional $4 \mathrm{~h}$. Subsequently, the cell culture medium was removed, and $100 \mu \mathrm{L} /$ well DMSO was added to dissolve formazan crystals in a humidified atmosphere overnight. Absorbance at $570 \mathrm{~nm}$ was measured using a 96-well microplate reader.

2.5. Crystal Violet Viability Assay. Crystal violet assay was conducted as described in [14]. Experimentally, iHUVEC cells were treated with drugs. At $24 \mathrm{~h}$ after treatment, cells were carefully washed with PBS and stained with $0.5 \%$ crystal violet formalin solution at room temperature for 20$30 \mathrm{~min}$. The stained cells were washed with tape water and air-dried for taking macrographic images. For quantitative measurement, the stained cells were dissolved in $10 \%$ acetic acid ( $1 \mathrm{~mL}$ per well for 12 -well plate) at room temperature for $20 \mathrm{~min}$ with shaking. Absorbance at $570-590 \mathrm{~nm}$ was measured.

2.6. Cell Adhesion Assay. For the cell adhesion model, HUVECs and 4T1 cells were used to study the adhesive ability between two different kinds of cell types. Briefly, HUVECs $\left(2 \times 10^{4}\right.$ each well) were grown to confluence on fibronectincoated wells of 96-well plates. The plates were blocked with Hank's balanced salt solution (HBSS) containing 1\% bovine serum albumin (BSA) (HBSS-BSA) for $30 \mathrm{~min}$ before the adhesion assay. BSA-coated wells serve as a negative control.

The 4T1 cells were trypsinized and suspended in HBSSBSA and then labeled with $10 \mu \mathrm{L}$ Hoechst 33258 for $30 \mathrm{~min}$ at $37^{\circ} \mathrm{C}$ followed by washing with HBSS-BSA. The labeled $4 \mathrm{~T} 1$ cells were then suspended in HBSS-BSA to a final density of $4.0 \times 10^{5}$ cells $/ \mathrm{mL}$, and different dosages of TET were added. Cell suspension $(100 \mu \mathrm{L} /$ well $)$ was incubated with HUVECs at $37^{\circ} \mathrm{C}$ for $30 \mathrm{~min}$. Cultures were carefully washed three times with PBS to remove nonadherent cells. Three random views were photographed in each well at 100x magnification with an inverted fluorescence microscope (Olympus Corporation, Japan). The image was analyzed with Image-Pro Plus 6 software (Media Cybernetics, USA).

2.7. Cell Migration Assay. A wound-healing model was used for evaluation of cell migration ability [15]. Cells treated with $0.1 \%$ DMSO were used as the vehicle control. Three random views along the scraped line were photographed in each well at $100 \mathrm{x}$ magnification before and after $10 \mathrm{~h}$ drug treatment 
with an inverted fluorescence microscope. The image was analyzed with Image-Pro Plus 6 software. Average scraped width of each well was measured and compared with control.

2.8. Boyden Chamber Transwell Cell Invasion Assay. Cell invasive ability was measured on a transwell system with a polycarbonate membrane $(8 \mu \mathrm{m}$ pores $)$ as previously described in [15]. The upper and lower sides of the membrane were precoated with $1: 30(\mathrm{v} / \mathrm{v})$ and $1: 100(\mathrm{v} / \mathrm{v})$ matrigel, respectively. The iHUVECs (50,000 cells) were seeded into culture inserts. Low-serum medium containing different concentrations of TET was added into the plate wells. After $12 \mathrm{~h}$, the inserts were washed with PBS; upper surface cells were removed by cotton swabs and the lower side was fixed in $3.7 \%$ paraformaldehyde. The invasive cells were then stained with propidium iodide (PI) and mounted on microscope slides. Images were captured at 200x magnification with an inverted fluorescence microscope. Invasive cells were quantified by Image-Pro Plus 6 software. The number of migrate cells per fields was determined by averaging nine randomly counted fields.

2.9. Tube Formation Assay. The effects of the drugs on HUVEC differentiation were examined by their in vitro tube formation ability on matrigel [15]. HUVECs were harvested and diluted to $2 \times 10^{5} \mathrm{cell} / \mathrm{mL}$ in low-serum medium $(0.5 \%$ FBS) containing $20 \mathrm{ng} / \mathrm{mL}$ Vascular Endothelial Growth Factor (VEGF) and different concentrations of drugs. The cells were then seeded onto $1: 1$ matrigel $(\mathrm{v} / \mathrm{v})$ coated 24 -well plates at $37^{\circ} \mathrm{C}$ for $8 \mathrm{~h}$. Cells treated with $0.1 \%$ DMSO were used as the vehicle control. The branch points of the capillary-like tubes were counted under light microscopy (100x field).

2.10. Cell Cycle Analysis by Flow Cytometry. Flow cytometry was used for quantitatively detecting the cell-cycle distribution [16]. Cells $\left(1 \times 10^{5} /\right.$ well $)$ were plated into 6 -well plates 1 day before treatment with TET at various concentrations. After treatment for 24 and $48 \mathrm{~h}$, cells were harvested, washed with PBS, fixed in cold $3.7 \%$ paraformaldehyde overnight at $4^{\circ} \mathrm{C}$ for at least $2 \mathrm{~h}$, and stained with $50 \mathrm{ng} / \mathrm{mL}$ PI in the presence of $200 \mu \mathrm{g} / \mathrm{mL}$ RNase A by incubation at $37^{\circ} \mathrm{C}$ for at least $30 \mathrm{~min}$. The stained cells were analyzed by flow cytometry (Becton-Dickinson). The red fluorescence (PE) representing the DNA content was collected through a $585 \mathrm{~nm}$ filter. Data were analyzed using Mod Fit LT 3.0 software.

2.11. Protein Extraction and Western Blotting Analysis. Western blotting was performed as previously described in [17]. Briefly, cells were collected and lysed in RAPI buffer. After treatment on ice for $30 \mathrm{~min}$, cell lysates were clarified by centrifugation at $11,419 \times \mathrm{g}$ for $20 \mathrm{~min}$ at $4^{\circ} \mathrm{C}$ to remove cell debris and the protein content was measured using a BCA protein assay kit (Beyotime, Jiangsu, China). Cleared total cell lysate was denatured by boiling, and aliquots of the lysates were loaded onto a $10 \%$ gradient SDS-PAGE. After electrophoretic separation, proteins were transferred to an Immobilon-P membrane. Membrane was blocked with
SuperBlock Blocking Buffer and probed with the primary antibody, anti-NF- $\kappa$ B (Santa Cruz Biotechnology, Santa Cruz, CA, USA), anti-ERK1/2, and p-ERK1/2 (Cell Signaling Technology, Vancouver, Canada), followed by incubation with a secondary antibody conjugated with biotin. Then the PVDF membrane was incubated with streptavidin HRP. The proteins of interest were detected by using SuperSignal West Pico Chemiluminescent Substrate kit.

2.12. Gelatin Zymography. Gelatin zymography was performed on $7.5 \%$ polyacrylamide gels containing $0.1 \%$ gelatin as previously described in [15]. Cells were treated as indicated in $0.5 \%$ FBS RPMI1640 (containing $20 \mathrm{ng} / \mathrm{mL}$ VEGF) for $24 \mathrm{~h}$. The cell culture medium was then centrifuged at $350 \times \mathrm{g}$ for $4 \mathrm{~min}$ at $4^{\circ} \mathrm{C}$, and the total protein of the supernatant was normalized with BCA protein assay kit. The supernatant was mixed with $5 x$ nonreducing sample buffer and loaded onto 10 well gels ( $20 \mu \mathrm{L} /$ sample), and electrophoresis was performed at $100 \mathrm{~V}$ for $1.25 \mathrm{~h}$. After electrophoresis, the gel was rinsed with $1 \mathrm{x}$ renaturing buffer for $1.5 \mathrm{~h}$ at room temperature. The buffer was then changed to $1 \mathrm{x}$ developing buffer and incubated for $48 \mathrm{~h}$ at $37^{\circ} \mathrm{C}$. Gelatin gel was stained with Coomassie blue and then destained with $10 \%$ acetic acid. The unstained bands correspond to the areas of gelatin digestion.

2.13. 4 T1 Tumor Bearing Mice Model. Female BALB/c mice (4 weeks old, 18-20 g, 10 mice per group) were used. Subconfluent 4T1-Luc cells were harvested and resuspended in PBS to a final density of $1 \times 10^{7}$ cells $/ \mathrm{mL}$. Before injection, cells were resuspended in PBS and analyzed by $0.4 \%$ trypan blue exclusion assay (viable cells, $>90 \%$ ). For cancer cell injection, approximately $5 \times 10^{5} 4 \mathrm{~T} 1$-Luc cells in $100 \mu \mathrm{L}$ of PBS were injected into the mammary fat pad (MFP) of each mouse using 27 gauge needles [18]. At $48 \mathrm{~h}$ after tumor cell injection, TET was administered at $10 \mathrm{mg} / \mathrm{kg}$ body weight to mice once every 2 days orally, and Dox was administered by intraperitoneal injection at $1 \mathrm{mg} / \mathrm{kg} / 2$ days to mice as a positive control.

2.14. Xenogen Bioluminescence Imaging. Small animal wholebody optical imaging was carried out as described previously in [9]. In brief, mice were anesthetized with isoflurane attached to a nose-cone mask equipped with Xenogen IVIS 200 imaging system (Caliper Life Sciences, Hopkinton, MA, USA) and subjected to imaging weekly after MFP injection.

For imaging, mice were injected intraperitoneally with D-luciferin sodium salt (Gold Biotechnology, St. Louis, MO, USA) at $100 \mathrm{mg} / \mathrm{kg}$ body weight in $0.1 \mathrm{~mL}$ of sterile PBS. Acquired images were obtained by superimposing the emitted light over the grayscale photographs of the animal. Quantitative analysis was done with Xenogen's Living Image V2.50.1 software as described previously in [14]. Animals were taken in vivo images for both untreated and treated groups and sacrificed after 4 weeks. Tumor, lung, and vascular samples were retrieved for histological examination.

2.15. High-Resolution Laser Doppler Perfusion Imaging. Microvascular blood flow was assessed by laser Doppler 
with a moorFLPI V2.1 software (Moor instruments Ltd, UK) $[19,20]$. Mouse hair was carefully removed and mice were anesthetized with isoflurane attached to a nose-cone mask. With a distance of $10 \mathrm{~cm}$ between the scanner and the skin surface, three examined areas $(1.4 * 1.4 \mathrm{~cm})$ were chosen so that tumor (Flux 1), adjacent healthy skin around tumor (Flux 2), and the heart of mice (Flux 3) were covered. The laser beam is reflected by the erythrocytes, which allows recording of the returning signal by a detector positioned in the scanner head and thus conversion to an electrical signal, proportional to the tissue perfusion. The underlying intensity of perfusion values is expressed on a scale of different colours extending from blue (low perfusion values) over green and yellow to red (highest perfusion values). The related perfusion values were calculated as follows: perfusion rate $($ tumor $)=F 1 / F 3 * 100 \%$; perfusion rate (vascular) $=$ F2/F3 * 100\%.

\subsection{Histological Evaluation and Immunohistochemical Stain-} ing. Retrieved tumor tissues were fixed in $10 \%$ formalin and embedded in paraffin. Serial sections of the embedded specimens were stained with hematoxylin and eosin. For immunohistochemical staining, slides were deparaffinized and then rehydrated in a graduated fashion [21]. The deparaffinized slides were subjected to antigen retrieval and probed with anti-ICAM-1, anti-HIF- $1 \alpha$, anti-integrin $\beta 5$, anti-ESM1, or anti-VEGF antibody (Santa Cruz Biotechnology) or isotype IgG control, followed by incubation with biotin secondary antibodies and streptavidin-horseradish peroxidase. The presence of the expected protein was visualized by DAB staining and examined under a microscope. Stains without the primary antibody were used as negative controls.

2.17. Statistical Analysis. Data were expressed as mean \pm S.D. Statistical significances between vehicle group versus drug treatment groups were determined by one-way analysis of variance. The $\mathrm{IC}_{50}$ of the TET was calculated by SPSS software. A value of $P<0.05$ was considered to be statistically significant.

\section{Results}

3.1. TET Exhibits a Significant Growth-Inhibitory Effect in HUVECs. To assess the antiangiogenic property of TET in vitro, we examined the inhibitory effects of TET on cell viability in HUVECs using MTT assay and crystal violet staining. As shown in Figures $1(\mathrm{a})$ and $1(\mathrm{~b})$, TET $(<10 \mu \mathrm{M})$ does not have any remarkable effect on HUVEC proliferation. However, TET can significantly inhibit cell viability at a much higher concentration with a half-maximal inhibition at $16.76 \mu \mathrm{M}$ (by MTT assay) or $29.31 \mu \mathrm{M}$ (by crystal violet staining assay). To examine the possible mechanism behind TET's inhibition effect on HUVEC's proliferation, we performed cell cycle analysis by FACS, and the result revealed that when HUVECs were treated with TET for $24 \mathrm{~h}$, TET in $10 \mu \mathrm{M}$ induced a depletion of cells in the $\mathrm{G}_{2}-\mathrm{M}$ phase, from $10.70 \%$ to $4.21 \%$, and a concomitant accumulation of cells in S phase, from $34.17 \%$ to $38.35 \%$. These data suggested that
TET could arrest endothelial cell proliferation. Furthermore, we examined the effects of TET on NF- $\kappa$ B, ERK1/2, and p-ERK1/2 expressions, and the results suggested that the inhibition of TET in HUVECs was related to the upregulation of NF- $\kappa \mathrm{B}$ and suppression of the phosphorylation of ERK1/2.

3.2. TET Inhibits Cell Adhesion, Cell Migration, and Cell Invasion of HUVECs. The antiadhesion ability of TET between 4T1 and HUVEC cells was investigated. As a result, TET could suppress the 4T1 and HUVEC adhesion after $30 \mathrm{~min}$ treatment (Figure 2(a)). The adhesive cells were decreased to 245 compared with 592 of the control group.

Moreover, the VEGF-induced migration of HUVEC cells was significantly suppressed by TET (Figure 2(b)). The migratory distance of HUVEC was significantly decreased by $10 \mu \mathrm{M}$ TET-contained medium when compared to that of control $(P<0.01)$. We further tested whether TET could affect chemotactic HUVECs invasion. Using the Boyden chamber transwell assay, we found that when HUVECs were treated with $10 \mu \mathrm{M}$ TET the numbers of migrated cells across the extracellular matrix protein-coated membranes significantly decreased (Figure 2(c)). Quantitatively, TET was shown to inhibit the numbers of migrated HCT116 cells by approximately $33 \%$ over that of the control treatment.

Notably, this inhibitory effect of TET on EC invasion was potentially related to the activity of proteinases (MMPs). To determine the effect of TET on the production of proteinases by HUVEC, culture supernatants were collected and subjected to gelatin zymography. As shown in Figure 2(d), the presence of proteinases (MMPs) digested the gelatincontaining gel and resulted in a clear band at $66 \mathrm{kDa}$, which was assigned to MMP-2, and TET reduced the gelatinolytic activities of secreted MMP-2 in a dose-dependent manner which corresponded to the inhibition of EC invasion.

In addition, we examined the expression of adhesion and invasion related factors, including integrin $\beta 5$, ESM-1, and ICAM-1 in vivo. The whole cell staining intensities of integrin $\beta 5$ and ESM-1 protein were markedly reduced in TET treated tumors, compared with those of the tumors from the control group (Figure 2(e)). On the contrary, the ICAM-1 level in cell was significantly decreased by TET treatment (Figure 2(e), the middle column). These results have demonstrated that TET can effectively inhibit EC adhesion, migration, and invasion through mechanisms of changing the integrin $\beta 5$, ESM-1, and ICAM-1 expressions.

3.3. TET Inhibits Tubeformation of HUVECs. The effect of TET on the capillary tube formation of ECs was examined. In the absence of VEGF, there was no tube network structure in ECs, whereas the addition of VEGF $(20 \mathrm{ng} / \mathrm{mL}$, positive control) induced the formation of tube or cordlike structure and tube network on GFR matrigel. Cultured with TET resulted in shorter and less blunted tubes of ECs than those of VEGF control group (Figure 3(a)). Quantitative measurements showed that TET caused an increase in mean tube branch point formation as compared to VEGF $20 \mathrm{ng} / \mathrm{mL}$ group (positive control) (Figure $3(\mathrm{~b})$ ). The number of branch 


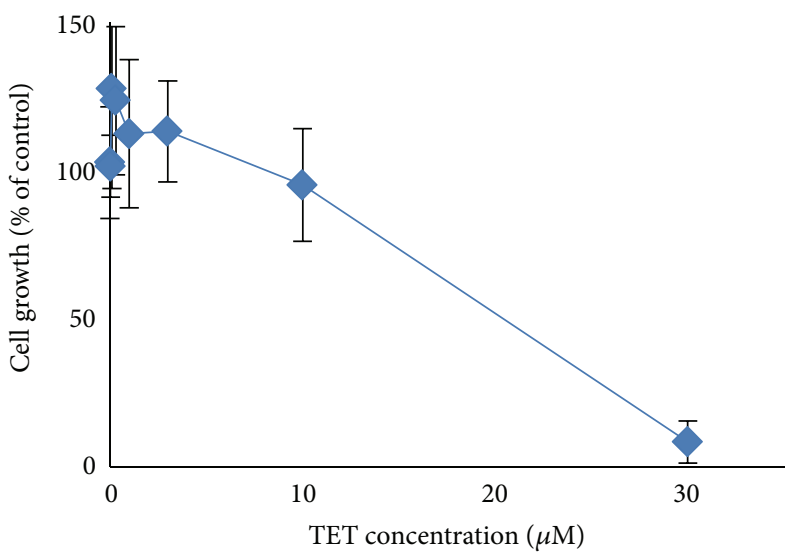

(a)

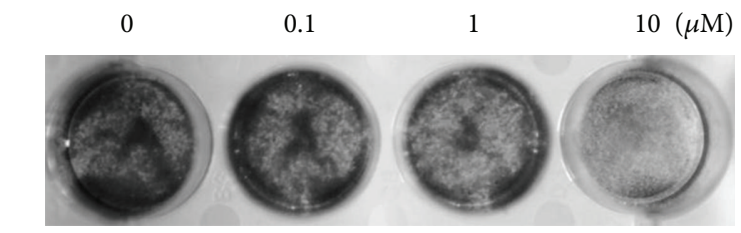

(b)

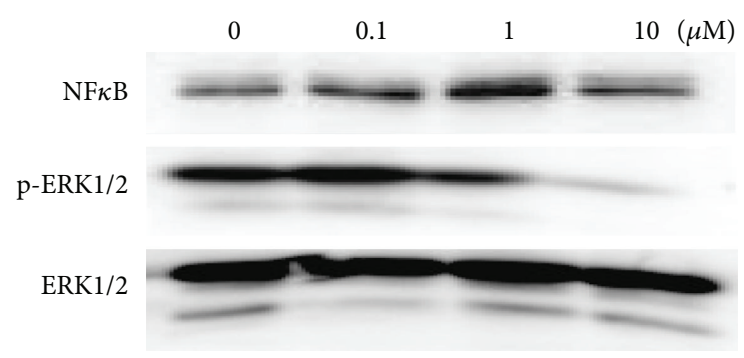

(d)
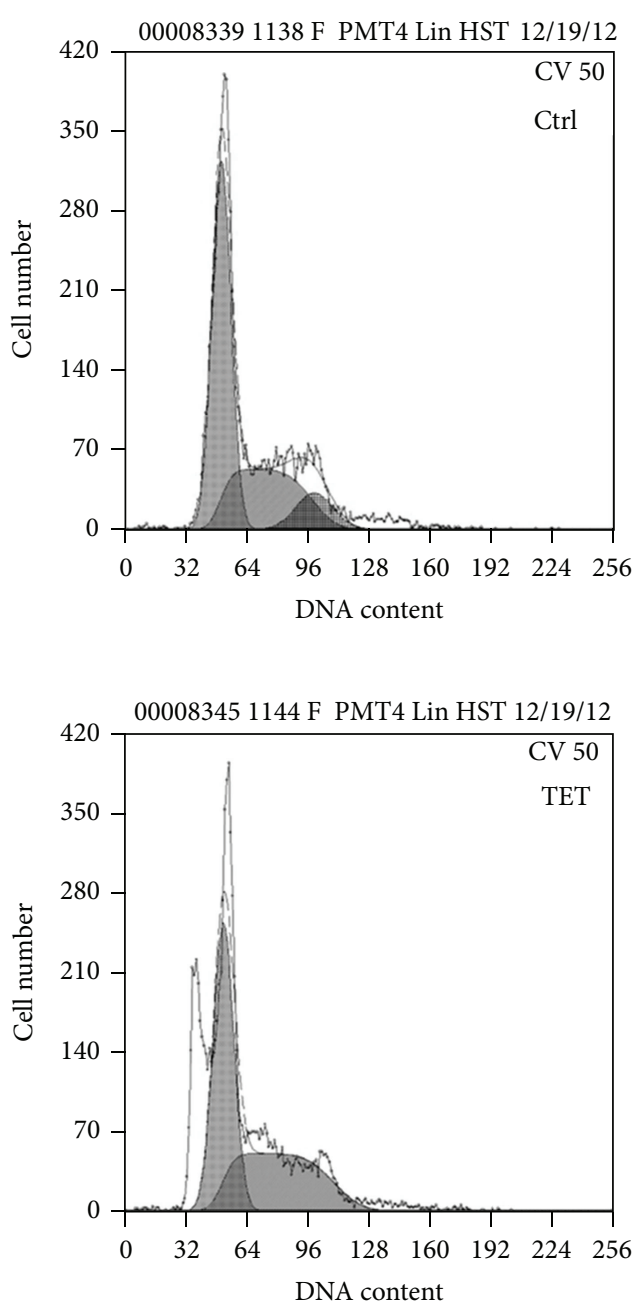

(c)

FIGURE 1: Antiproliferative activity of TET in HUVECs. (a) MTT assay. Subconfluent HUVECs were treated with indicated concentrations of TET for $48 \mathrm{~h}$. The cells were then subjected to MTT assay. Each assay condition was done in triplicate $(n=12)$. (b) Crystal violet assay. HUVECs were treated with TET at the indicated concentrations for $24 \mathrm{~h}$. The cells were subjected to crystal violet assay as described in Section 2. (c) Cell cycle analysis. Cell cycle distribution of HUVECs was analyzed by flowcytometry. Cells were treated with $1 \mu \mathrm{M}$ TET for $24 \mathrm{~h}$ and fixed, and then nuclear DNA was labeled with PI. Histogram display DNA content ( $x$-axis [PE-A]: PI-fluorescence) versus cell counts ( $y$-axis). (d) ERK, p-ERK, and NF $\kappa$ B expressions in HUVECs. The expression of ERK, p-ERK, and NF $\kappa$ B was detected by western blot.

was reduced from 15.7 per area in the control group to 3.6 in the group treatment with TET $(10 \mu \mathrm{M})(P<0.01)$.

\subsection{TET Inhibits In Vivo Tumor Metastasis in Mouse Breast} Tumor Model. We next investigated the in vivo antimetastatic activity of TET using a mouse breast cancer model. Briefly, exponentially growing firefly luciferase-tagged 4T1 cells were injected into the MFP of BALB/c mice, and TET was orally administered (10 mg/kg body weight, once every two days). As shown in Figure 4(a), the Dox and TET treatment groups exhibited significantly decreased Xenogen imaging signal in lung, when compared with the control group four weeks after treatment. At sacrifice, lung metastases were counted. In keeping with the in vitro data, the number of metastasis sites on the lung surface was remarkably decreased by TET, from 6.2 to 2.6 for each mouse, rather than that of Dox treated mouse (Figures 4(b) and 4(c)). Moreover, histological analysis ( $\mathrm{H} \& \mathrm{E}$ staining) indicated that TET treatment group exhibited a decreased metastatic tumor mass in lung (Figure 4(d)).

3.5. TET Inhibits In Vivo Angiogenesis in $4 T 1$ Tumor Bearing Mice. The effect of TET on angiogenesis in vivo was also examined in the animal model. As shown in Figure 5(a), four weeks after MFP injection of $4 \mathrm{~T} 1$ cells into mice, the diameter of the blood vessels in the tumor implanted side is increased, rather than in the other side, but TET could significantly inhibit the increase of the blood vessel diameter from control levels. Consistent with gross observations, solid tumor sections further indicated that TET inhibited neovascularization in tumor mass in vivo, and the average 


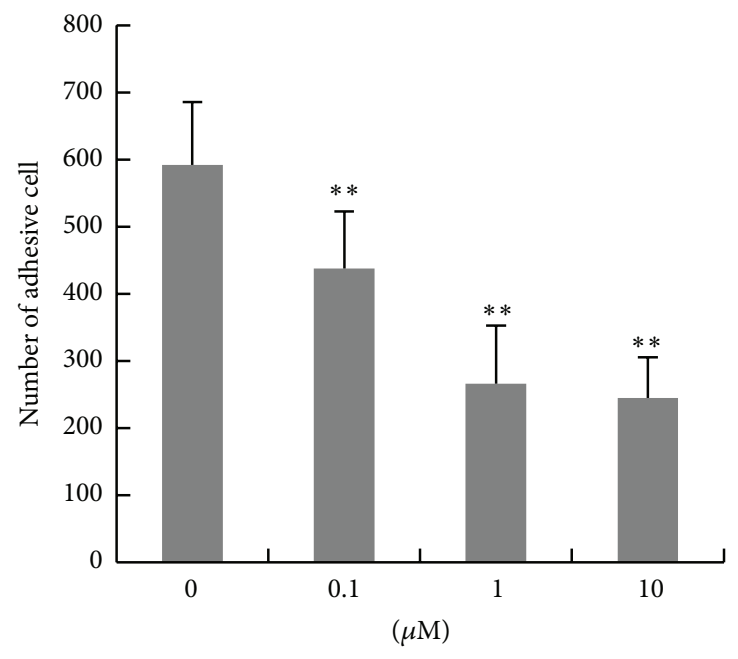

(a)

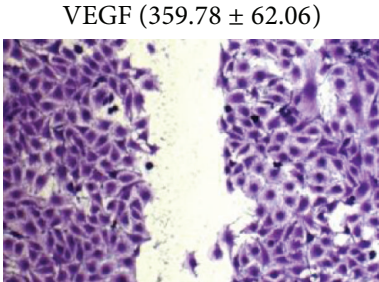

TET $1 \mu \mathrm{M}(605.41 \pm 64.8)$

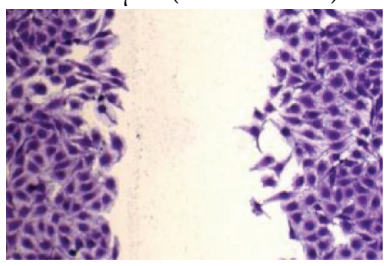

(b)
TET $0.1 \mu \mathrm{M}(470.72+83.54)$

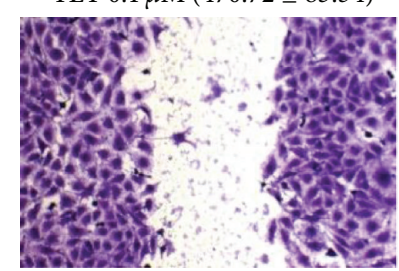

TET $10 \mu \mathrm{M}(540.48 \pm 75.29)$
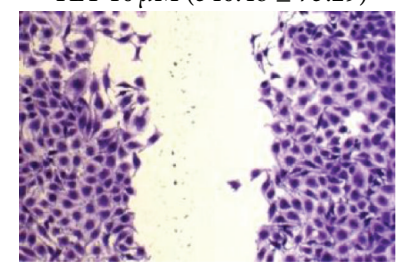

TET $(10 \mu \mathrm{M})$

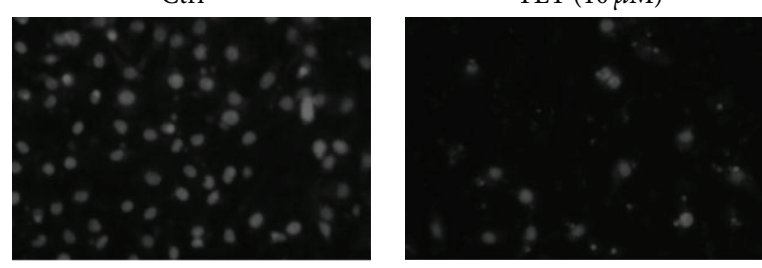

(c)

ESM-1
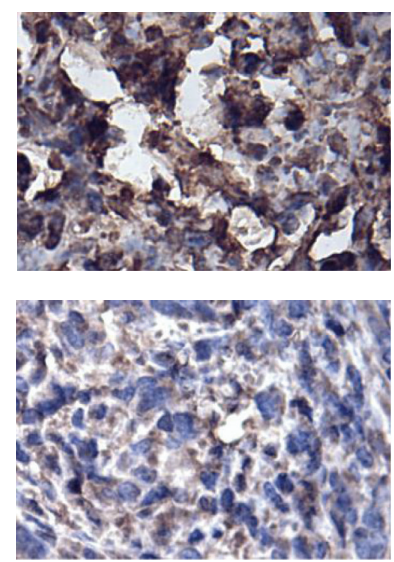

ICAM-1
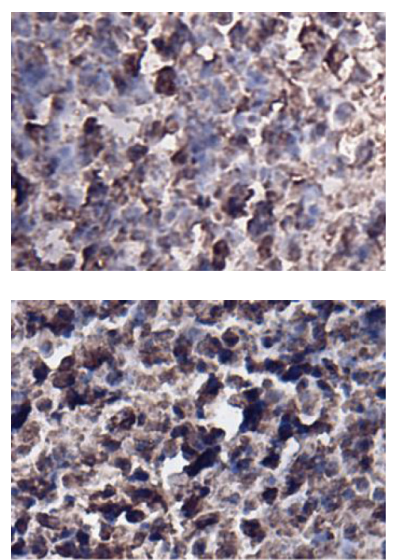

$\begin{array}{llllll}\mathrm{M} & 0 & 0.1 & 1 & 10 & (\mu \mathrm{M})\end{array}$

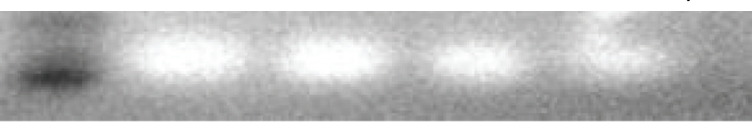

(d)

ITG $\beta 5$
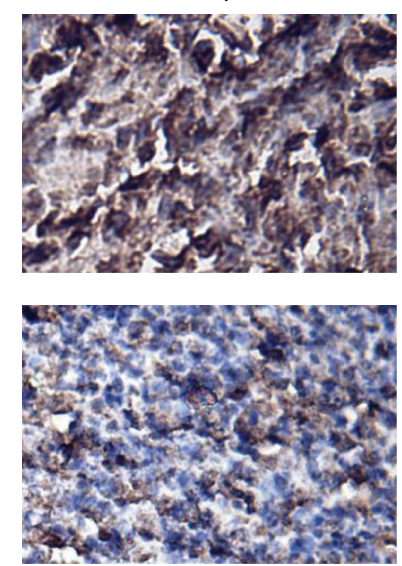

(e)

FIgURE 2: TET inhibits cell adhesion (a), migration (b), and invasion (c) in HUVECs. (a) Cell adhesion assay. HUVECs and 4T1 cells were used to study the cell adhesive ability. ${ }^{* *} P<0.01$ versus vehicle control. (b) Cell migration assay. Wound-healing model was used for evaluation of cell migration ability. Three random views were photographed along the scraped line in each well at 100x magnification. (c) Cell invasion assay. Cell invasive ability was measured with a transwell system with a polycarbonate membrane ( $8 \mu \mathrm{m}$ pores). Images were captured at $200 \mathrm{x}$ magnification. (d) Effects of TET on secretion of matrix metalloproteinase-2 (MMP-2). Gelatin zymography was carried out in an SDS-PAGE gel that contained $0.1 \%$ gelatin. (e) Effect of TET on ESM-1, ICAM-1, and integrin $\beta 5$ expressions in tumor tissues. 4 T1 tumor bearing mice (top panel) and TET treated tumor bearing mice (bottom panel) (magnification $\times 400)$.

number of new capillaries blood vessel in control group was more than that in TET treated group (Figure 5(b)). These results demonstrated that TET is a potent inhibitor of vascularization and angiogenesis.

We sought to further investigate the mechanism behind the TET mediated inhibition of angiogenesis activity. As shown in Figure 5(c), we examined the expression of metastatic and angiogenic related proteins, including VEGF and HIF- $1 \alpha$ in vivo. The whole cell and nuclear staining intensities of VEGF and HIF-1 $\alpha$ were markedly reduced in TET treated tumors, compared with those of the control group. 


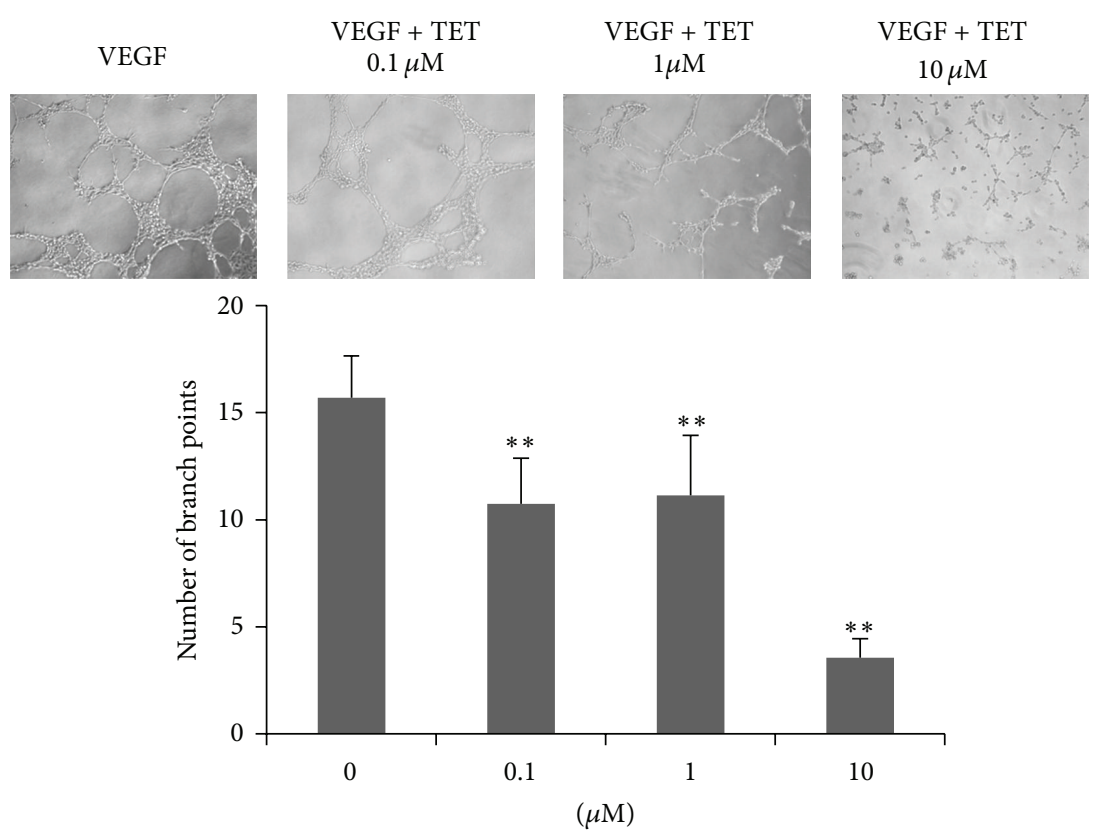

FIgURE 3: TET inhibits tube formation in HUVECs. The effects of TET on HUVEC differentiation were examined by their in vitro tube formation ability on matrigel. The branch points of the capillary-like tubes were counted under light microscopy (magnification 100x). Each value represents the mean \pm S.D. of triplicate samples in each case. ${ }^{* *} P<0.01$ versus the VEGF-only group.

Lastly, to investigate the blood perfusion change of TET treated tumor bearing mice in tumor surface and skin around tumor area, we took advantage of the laser Doppler perfusion imaging (LDPI) to measure the local perfusion pattern. In the LDPI, the related perfusion of the mice abdomen was decreased after tumor being implanted for 3 weeks, especially the tumor site (Figure 6(a)). The related perfusion of tumor site (Flux 1) in TET group was decreased to $49 \%$ lower than that in healthy mice, but with no difference from that of the model group. It suggested that TET could slightly improve the necrosis of tumor 4 weeks after TET administration (Figure 6(b)). However, the related perfusion around tumor site (Flux 2) in TET group was significantly decreased to $82.54 \%$ of the model group $(P<0.05)$, which indicated that TET could markedly decrease the local blood perfusion of tumor 3 weeks after TET administration (Figure 6(c)) and suggested that the tumor angiogenesis was suppressed by TET treatment.

Taken together, these in vivo results strongly suggest that TET may inhibit the tumor metastasis of breast cancer, possibly by reducing angiogenesis activity and related protein level of breast cancer cells, although further investigation is required.

\section{Discussion}

The growth and progression of solid tumors are usually limited by the nutrient supply for tumor. Thus, the blockage of microvessels formation and local blood perfusion in tumor might be useful in cancer therapy. Recently, more than 20 antiangiogenic drugs including TNP-470, thalidomide, and endostatin are subjected to different phases of clinical trials.
In addition, phytochemicals such as resveratrol, salvianolic acid $\mathrm{B}$, and ginseng saponins were found to exert inhibitory effect on the vascularization [22].

TET has been shown to exhibit anticancer activity in many in vivo models $[5,9,10]$. TET-treated mice $(10 \mathrm{mg} / \mathrm{kg} /$ day $)$ have fewer metastases than vehicle treated mice, and no acute toxicity or obvious body weight changes [23]. Recent studies showed that TET induces cell cycle arrest and also induces apoptosis in many human cancer cells. In our previous study, we found that inhibition of Wnt/betacatenin signaling might contribute to the anticancer effects of TET [9]. Nonetheless, it is conceivable that other signaling pathways may also participate in TET's anticancer activity. For example, activation of glycogen synthase kinase $3 \beta$ (GSK$3 \beta)$, generation of ROS, activation of p38 mitogen-activated protein kinase (p38 MAPK), and upregulation of p53, p21, p27, and Fas might contribute to the anticancer effects of TET [5, 24-29].

As mentioned previously, TET exhibits significant anticancer activity both in vitro and in vivo, as well as its inhibitory effect on tumor metastasis and angiogenesis. Chen et al. found that TET inhibits the expression of VEGF in glioma cells, has cytotoxic effect on ECV304 HUVECs, and suppresses in vivo angiogenesis in rat [10]. However, the tumor related angiosuppressive property of TET and the molecular mechanism that underlies its activity are not fully understood. In the present study, we used a new LDPI method combined with different angiogenesis assays that are related to proliferation, adhesion, migration, invasion, and tube formation of EC during angiogenic process, to assess the angiosuppressive activity of TET. 


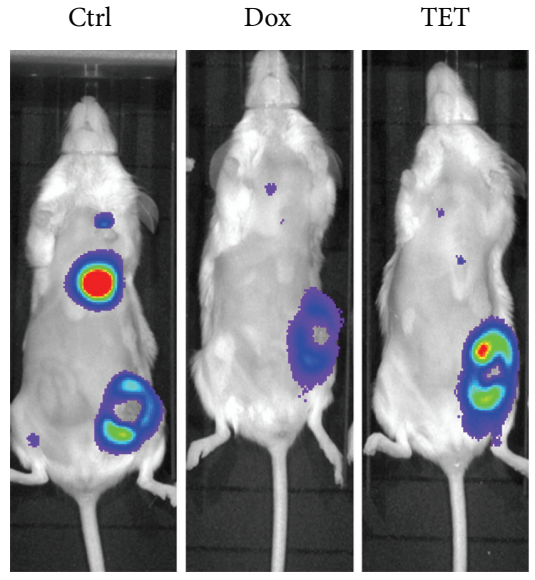

(a)

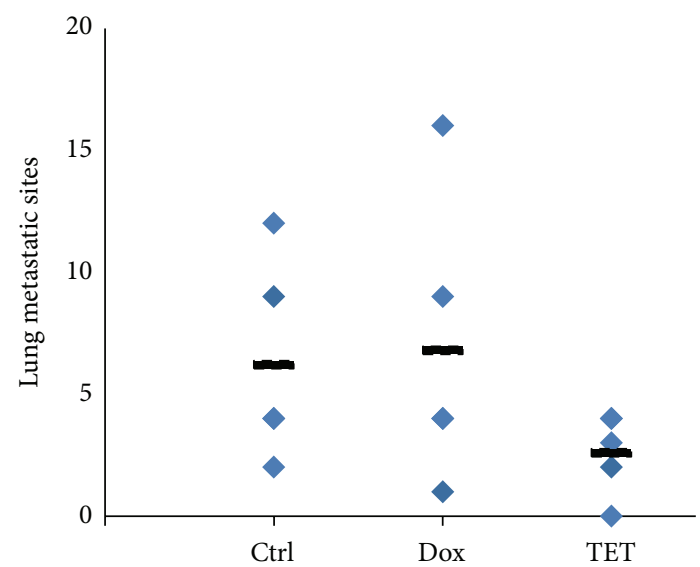

(b)

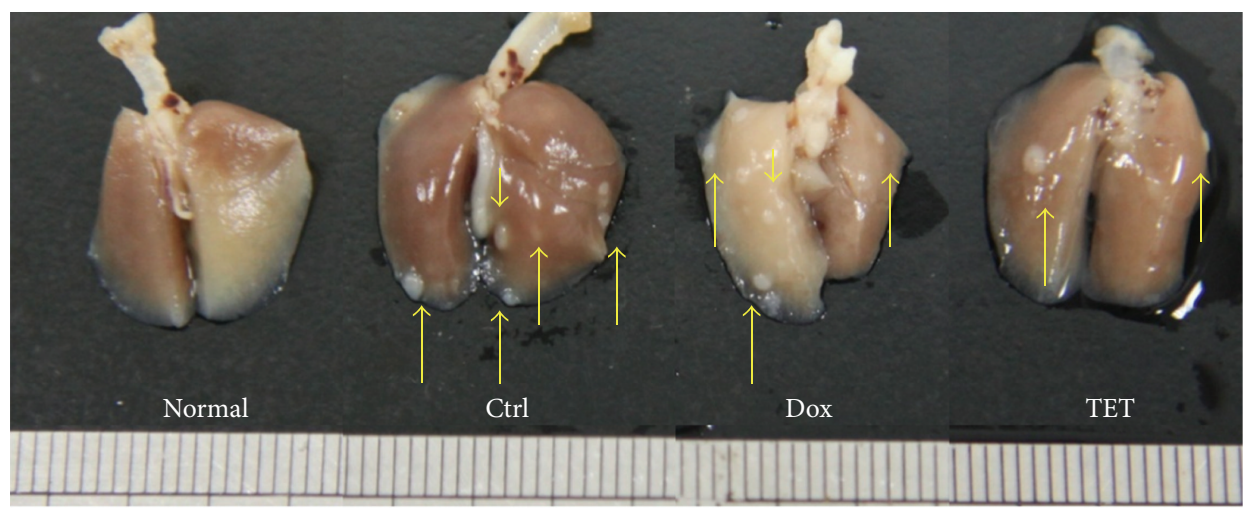

(c)

Ctrl

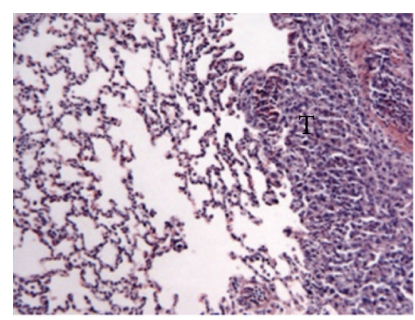

Dox

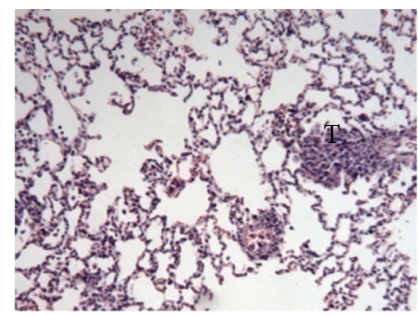

TET

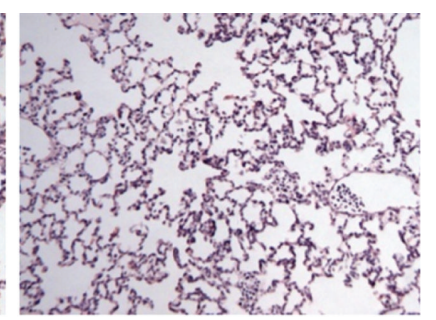

(d)

FIGURE 4: TET inhibits in vivo tumor metastasis in mouse breast cancer model. (a) Bioluminescence imaging of TET treated tumor bearing mice. Firefly luciferase-tagged 4T1 cells were injected into the MFP of BALB/c mice and TET was orally administered (10 mg/kg body weight, once every two days); images were obtained by using IVIS 200 imaging system. Representative Xenogen imaging results at week 4 are shown. (b) TET reduced tumor lung metastasis sites in 4T1 tumor-bearing mice. Picture shows the number of lung metastasis sites on the lung surface for each mouse. The black line shows the average number of metastasis sites for each group. (c) Photograph of pulmonary metastases. Animals were sacrificed after 4 weeks, and the lungs were dissected and photographed. (d) Histological examination of lung samples. Lung tissues sections were stained with H\&E stain, and photographs were made under a microscope at a magnification of 400x (T: tumor).

Results from the present study demonstrated that TET exerted inhibitory effect on proliferation, adhesion, and capillary tube formation of ECs in a dose-dependent manner. Interestingly, the blood perfusion of the periphery of tumors was significantly reduced by TET treatment. Owing to that the blood perfusion is usually proportional to the body's blood vessels density [30], this result implied that the antimetastasis effect of TET was passably related to the angiosuppressive activity. Similar cases were also observed in the tumor mass of TET treated 4T1-tumor bearing mice. TET was found to effectively suppress the formation of micro vessels in tumor. Furthermore, since tube formation of HUVEC involves EC attachment, migration, and production of ECM degrading enzymes, data in the presented paper indicated that all these steps were interfered by TET and resulted in the attenuation of angiogenesis in vitro and in vivo. Thus, TET may be useful in cancer metastasis by acting as a specific and effective angiosuppressive agent. 
Normal

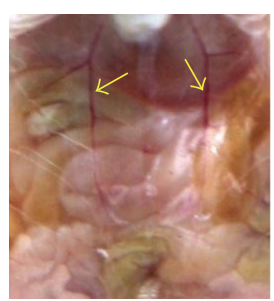

Ctrl



VEGF
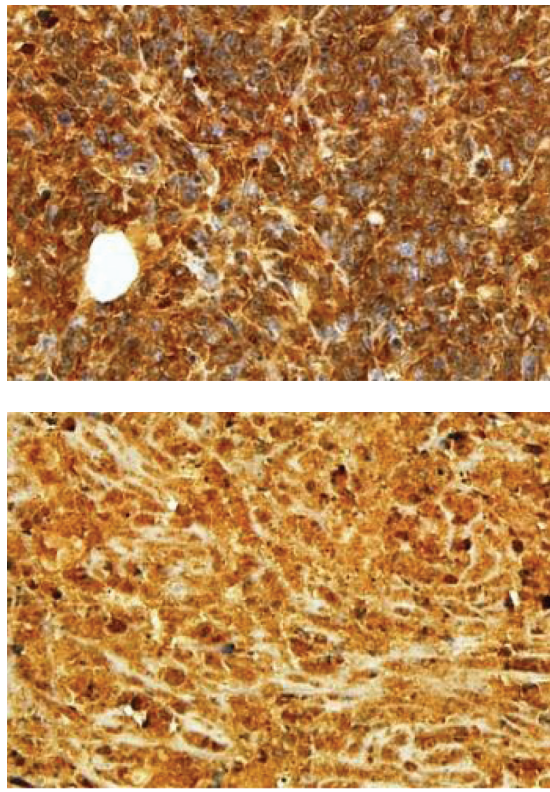

Dox

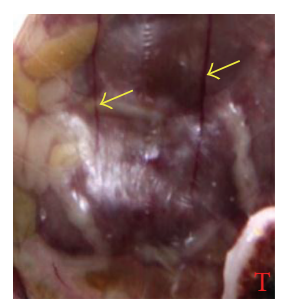

TET
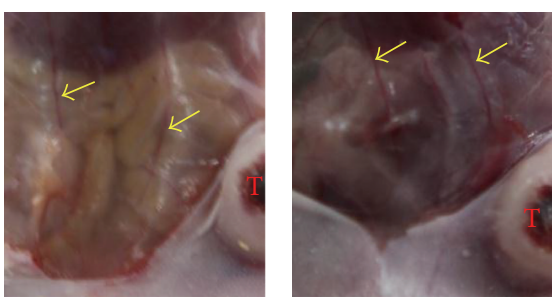

TET

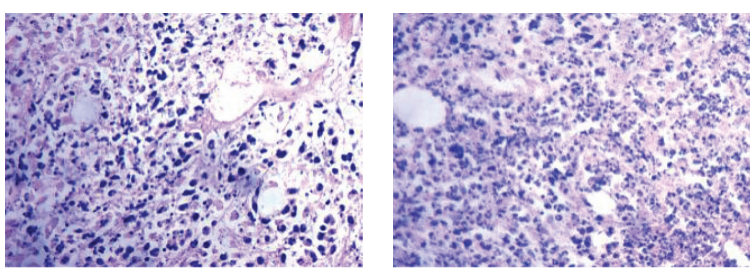

(b)
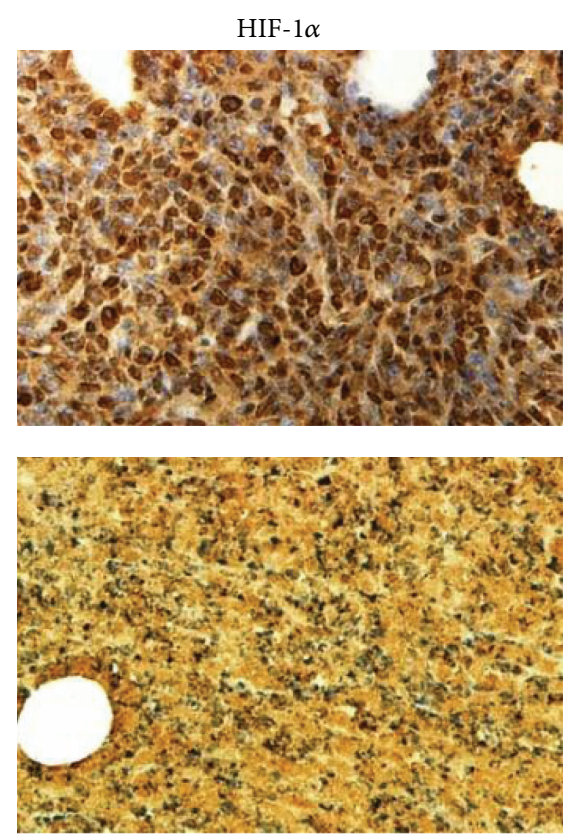

(c)

FIGURE 5: TET suppressed tumor angiogenesis in $4 \mathrm{~T} 1$ tumor bearing mice. (a) The blood vessel diameter of TET treated tumor bearing mice. Picture shows the diameter of blood vessel on mice abdomen for each mouse. (b) Hematoxylin \& Eosin staining of tumor tissues. Retrieved tumor samples were fixed, embedded, and subjected to H\&E staining. Representative images are shown (magnification, $\times 400$ ). (c) VEGF and HIF-1 $\alpha$ expressions in tumor tissues. Representative staining results are shown: 4T1 tumor-bearing BALB/c mice (top panel) and TET treated mice (bottom panel) (magnification, $\times 400$ ).

We investigated the molecular mechanism underlying the antivascularization activity of TET in breast cancer. TET treated group exhibited a decreased level of VEGF, HIF- $1 \alpha$, ESM- 1 and Integrin $\beta 5$ protein but upregulation of ICAM-1 level. These in vivo results strongly suggest that the inhibitory effect of TET on breast cancer metastasis may be at least in part mediated by inhibiting tumor angiogenesis factors (VEGF and HIF-1 $\alpha$ ) or regulating adhesion factors (Integrin $\beta 5$, ESM-1, and ICAM-1), although further investigation is required.
One of the key cell signaling pathways involved in cancer tumorigenesis and metastasis is the hypoxic pathway. As we know, HIF- $1 \alpha$ binds to HREs and induces subsequent expression of genes encoding angiogenic factors, such as VEGF and MMPs, leading to angiogenesis [31]. TET could reduce the expression of HIF- $1 \alpha$ and then decrease VEGF level and MMPs activity. It is generally believed that VEGF could activate ECs; activated ECs produce many types of enzymes such as matrix metalloproteinases (MMPs) that break down the stroma and ECM proteins [32]. This is 

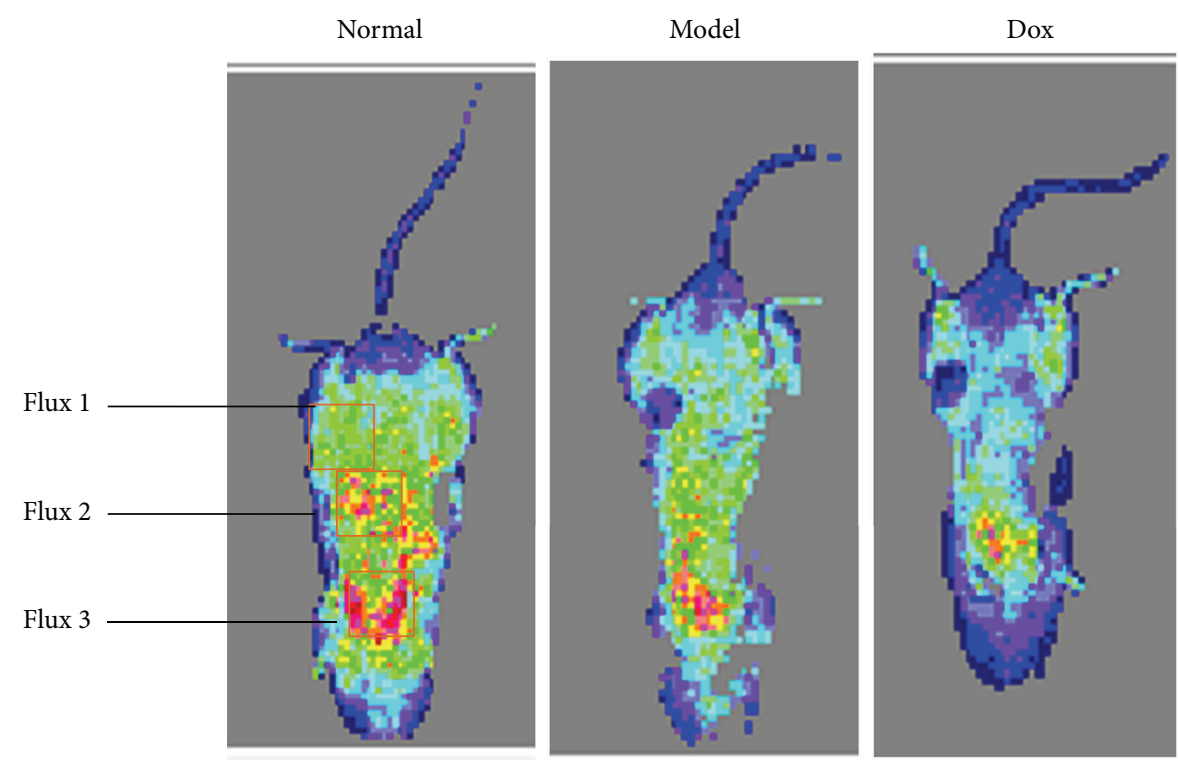

(a)

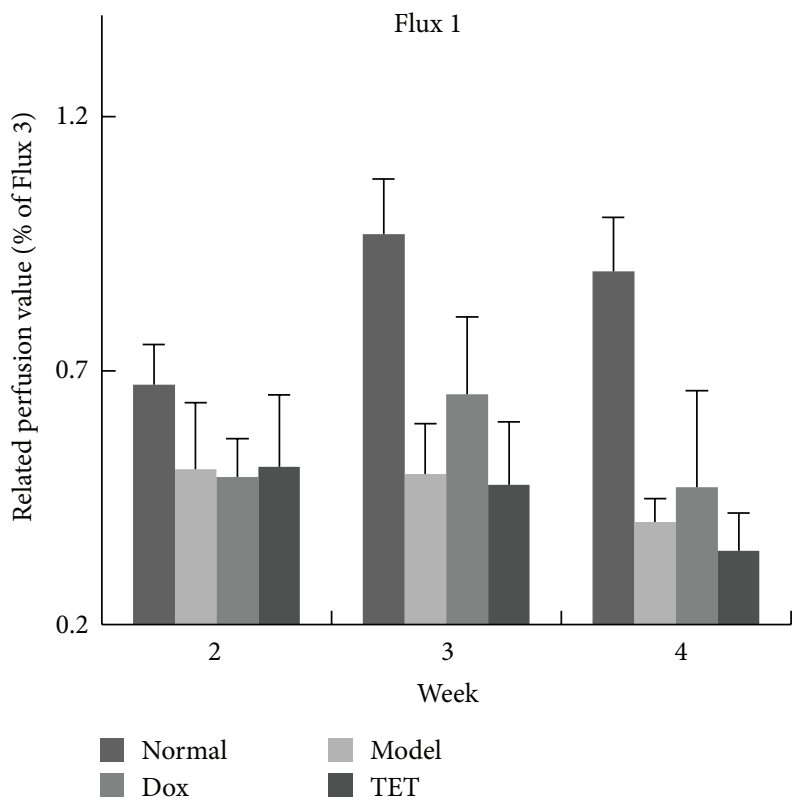

(b)

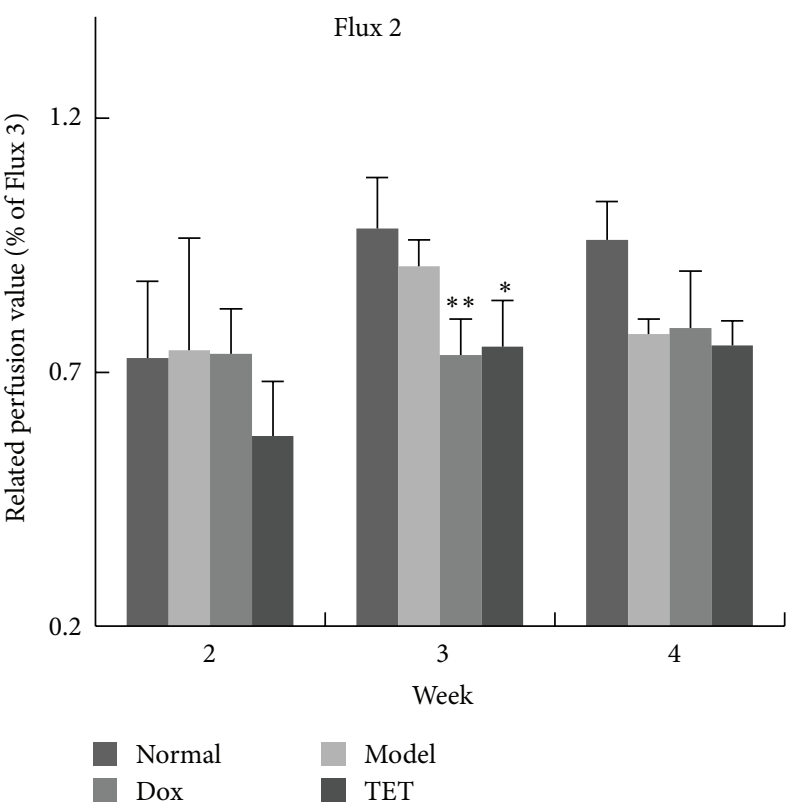

(c)

FIGURE 6: TET inhibits in vivo blood perfusion in $4 \mathrm{~T} 1$ tumor bearing mice. (a) In vivo LDPI imaging of mice. TET was orally administered $(10 \mathrm{mg} / \mathrm{kg}$, once/2 days). With a distance of $10 \mathrm{~cm}$ between the scanner and the skin surface, three examined areas $(1.4 * 1.4 \mathrm{~cm}) \mathrm{covered}$ the tumor (Flux 1), adjacent healthy skin around tumor (Flux 2), and the heart of mice (Flux 3). (b and c) Quantitative analysis of microvascular blood perfusion in mice. Related perfusion $($ Flux 1$)=F 1 / F 3 * 100 \%$; related perfusion (Flux 2$)=F 2 / F 3 * 100 \%$. ${ }^{*} P<0.05,{ }^{* *} P<0.01$ versus vehicle control.

the critical step in angiogenesis as well as metastasis. The invasion assay involving the migration of HUVEC through ECM (matrigel) demonstrated that TET could reduce the chemoinvasive ability of EC by reducing the gelatinases activities of the cell culture medium. These noteworthy results indicated that the angiosuppressive effect of TET could be possibly due to the reduction of HIF-1 $\alpha$ and/or VEGF expression, as well as MMPs activities.

Recently, several research groups have tried to identify cell adhesion suppressors which could inhibit cancer metastasis by blocking the lodging in blood vessels in the distant organs of disseminated cancer cells or cell clusters [33]. Integrins are important mediators of the malignant phenotype during oncogenic transformation [34]. Breast carcinoma cells express high levels of integrin $\beta 5$. BianchiSmiraglia et al. indicated that cells deficient in integrin $\beta 5$ have lower migration and proliferative capacities, and ERK signaling pathway plays an important role in the function of integrin $\beta 5$ in cancer [35]. Our results show that the expression of integrin $\beta 5$ was decreased by TET treatment 


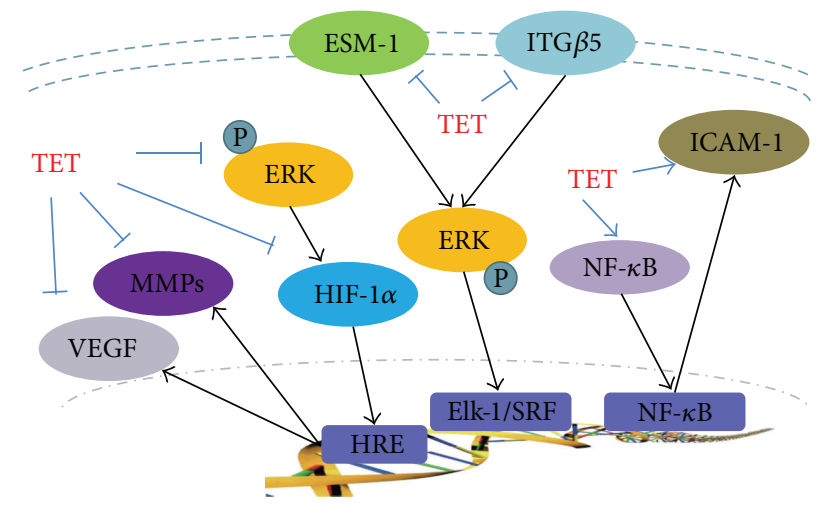

FIGURE 7: The influence of TET on the angiogenesis and metastasis related factors.

and accompanied by a reduced phosphorylated-ERK level. On the other hand, the adhesion factor ESM-1, which could inhibit leukocyte adhesion and migration through the endothelium, was increased in tissue and serum from colorectal cancer patients and ESM-1 silencing decreased cell survival, migration, and invasion and modulated cell cycle progression in hepatocellular carcinoma [36, 37]. We notified that TET also decreases ESM-1 expression in breast cancer cells, which leads to an inhibition effect of tumor metastasis. Taken together, TET suppressed the integrin $\beta 5$ and ESM-1 expressions, then depressed the activation of ERK, and regulated cellular proliferation, adhesion, and survival in ECs.

Another important adhesive factor involved in TET's angiosuppressive effect is ICAM-1; several researches showed that ICAM-1 synthesis in ECs is regulated by activation of p38 and NF- $\kappa$ B $[38,39]$, and ICAM-1 could induce cell adhesion by active ERK, JNK, and p38 pathways [40, 41]. Our IHC results present an upregulation of ICAM-1 in tumor tissue, along with the increasing of NF- $\kappa \mathrm{B}$, and suggested that the promotion effect of TET on NF- $\kappa$ B and ICAM-1 expressions is closely related to its antiangiogenesis effects. Taken together, as shown in Figure 7, our studies indicate that TET is a potential inhibitor of tumor angiogenesis and metastasis by targeting the angiogenesis and metastasis related factors.

\section{Conflict of Interests}

The authors declare that there is no conflict of interests.

\section{Authors' Contribution}

J.-L. Gao and X. Ji contributed equally to the work.

\section{Acknowledgments}

The reported work was supported by China National Natural Science Foundation (no. 81102852, 81228024), the Zhejiang Provincial Key Laboratory Project (no. 2012E10002), and the Innovation Group Project of Zhejiang Chinese Medical University.

\section{References}

[1] J. J. Lu, J. L. Bao, X. P. Chen, M. Huang, and Y. T. Wang, "Alkaloids isolated from natural herbs as the anticancer agents," Evidence-Based Complementary and Alternative Medicine, vol. 2012, Article ID 485042, 12 pages, 2012.

[2] L. W. Fu, Y. M. Zhang, Y. J. Liang, X. P. Yang, and Q. C. Pan, "The multidrug resistance of tumour cells was reversed by tetrandrine in vitro and in xenografts derived from human breast adenocarcinoma MCF-7/adr cells," European Journal of Cancer, vol. 38, no. 3, pp. 418-426, 2002.

[3] T. H. Wang, J. Y. Wan, X. Gong, H. Z. Li, and Y. Cheng, "Tetrandrine enhances cytotoxicity of cisplatin in human drugresistant esophageal squamous carcinoma cells by inhibition of multidrug resistance-associated protein 1," Oncology Reports, vol. 28, no. 5, pp. 1681-1686, 2012.

[4] Y. L. Lai, Y. J. Chen, T. Y. Wu et al., "Induction of apoptosis in human leukemic U937 cells by tetrandrine," Anti-Cancer Drugs, vol. 9, no. 1, pp. 77-81, 1998.

[5] J. M. Wu, Y. Chen, J. C. Chen, T. Y. Lin, and S. H. Tseng, "Tetrandrine induces apoptosis and growth suppression of colon cancer cells in mice," Cancer Letters, vol. 287, no. 2, pp. 187-195, 2010.

[6] X. Li, B. Su, R. Liu, D. Wu, and D. He, “Tetrandrine induces apoptosis and triggers caspase cascade in human bladder cancer cells," Journal of Surgical Research, vol. 166, no. 1, pp. e45-e51, 2011.

[7] L. T. Ng, L. C. Chiang, Y. T. Lin, and C. C. Lin, "Antiproliferative and apoptotic effects of tetrandrine on different human hepatoma cell lines," American Journal of Chinese Medicine, vol. 34, no. 1, pp. 125-135, 2006.

[8] J. H. Lee, G. H. Kang, K. C. Kim et al., "Tetrandrine-induced cell cycle arrest and apoptosis in A549 human lung carcinoma cells," International Journal of Oncology, vol. 21, no. 6, pp. 1239-1244, 2002.

[9] B. C. He, J. L. Gao, B. Q. Zhang et al., "Tetrandrine inhibits Wnt/ $\beta$-catenin signaling and suppresses tumor growth of human colorectal cancer," Molecular Pharmacology, vol. 79, no. 2, pp. 211-219, 2011.

[10] Y. Chen, J. C. Chen, and S. H. Tseng, "Tetrandrine suppresses tumor growth and angiogenesis of gliomas in rats," International Journal of Cancer, vol. 124, no. 10, pp. 2260-2269, 2009.

[11] E. Huang, Y. Bi, W. Jiang et al., "Conditionally immortalized mouse embryonic fibroblasts retain proliferative activity without compromising multipotent differentiation potential," PLoS One, vol. 7, no. 2, Article ID e32428, 2012.

[12] Y. Su, X. Luo, B. C. He et al., "Establishment and characterization of a new highly metastatic human osteosarcoma cell line," Clinical and Experimental Metastasis, vol. 26, no. 7, pp. 599-610, 2009.

[13] J. L. Gao, T. C. He, Y. B. Li, and Y. T. Wang, "A traditional chinese medicine formulation consisting of Rhizoma corydalis and Rhizoma curcumae exerts synergistic anti-tumor activity," Oncology Reports, vol. 22, no. 5, pp. 1077-1083, 2009.

[14] B. C. He, J. L. Gao, X. Luo et al., "Ginsenoside Rg3 inhibits colorectal tumor growth through the down-regulation of Wnt/ $\beta$ catenin signaling," International Journal of Oncology, vol. 38, no. 2, pp. 437-445, 2011.

[15] J. L. Gao, J. M. Shi, S. M. Y. Lee, Q. W. Zhang, and Y. T. Wang, "Angiogenic pathway inhibition of Corydalis yanhusuo and berberine in human umbilical vein endothelial cells," Oncology Research, vol. 17, no. 11-12, pp. 519-526, 2009. 
[16] Y. W. Liu, J. L. Gao, J. Guan, Z. M. Qian, K. Feng, and S. P. Li, "Evaluation of antiproliferative activities and action mechanisms of extracts from two species of ganoderma on tumor cell lines," Journal of Agricultural and Food Chemistry, vol. 57, no. 8, pp. 3087-3093, 2009.

[17] J. L. Gao, J. M. Shi, K. He et al., "Yanhusuo extract inhibits metastasis of breast cancer cells by modulating mitogenactivated protein kinase signaling pathways," Oncology Reports, vol. 20, no. 4, pp. 819-824, 2008.

[18] S. A. DuPré, D. Redelman, and K. W. Hunter Jr., “The mouse mammary carcinoma 4T1: characterization of the cellular landscape of primary tumours and metastatic tumour foci," International Journal of Experimental Pathology, vol. 88, no. 5, pp. 351-360, 2007.

[19] M. Stücker, M. Esser, M. Hoffmann et al., "High-resolution laser Doppler perfusion imaging aids in differentiating between benign and malignant melanocytic skin tumours," Acta Dermato-Venereologica, vol. 82, no. 1, pp. 25-29, 2002.

[20] D. Esser, E. Oosterink, J. op 't Roodt et al., "Vascular and inflammatory high fat meal responses in young healthy men, a discriminative role of IL-8 observed in a randomized trial," PLoS One, vol. 8, no. 2, Article ID e53474, 2013.

[21] L. Chen, W. Jiang, J. Huang et al., "Insulin-like growth factor 2 (IGF-2) potentiates BMP-9-induced osteogenic differentiation and bone formation," Journal of Bone and Mineral Research, vol. 25, no. 11, pp. 2447-2459, 2010.

[22] T. P. Fan, J. C. Yeh, K. W. Leung, P. Y. K. Yue, and R. N. S. Wong, "Angiogenesis: from plants to blood vessels", Trends in Pharmacological Sciences, vol. 27, no. 6, pp. 297-309, 2006.

[23] K. H. Chang, H. F. Liao, H. H. Chang et al., "Inhibitory effect of tetrandrine on pulmonary metastases in CT26 colorectal adenocarcinoma-bearing BALB/c mice," American Journal of Chinese Medicine, vol. 32, no. 6, pp. 863-872, 2004.

[24] P. L. Kuo and C. C. Lin, "Tetrandrine-induced cell cycle arrest and apoptosis in Hep G2 cells," Life Sciences, vol. 73, no. 2, pp. 243-252, 2003.

[25] L. H. Meng, H. Zhang, L. Hayward, H. Takemura, R. G. Shao, and Y. Pommier, "Tetrandrine induces early G1 arrest in human colon carcinoma cells by down-regulating the activity and inducing the degradation of G 1-S-specific cyclin-dependent kinases and by inducing p53 and p21Cip1," Cancer Research, vol. 64, no. 24, pp. 9086-9092, 2004.

[26] J. A. McCubrey, J. Bäsecke, M. Cervello, A. M. Martelli, and R. A. Franklin, "GSK-3 $\beta$ is a critical mediator of tetrandrine induced cell cycle arrest and cytotoxicity," Cancer Biology and Therapy, vol. 7, no. 7, p. 1079, 2008.

[27] X. L. Chen, K. H. Ren, H. W. He, and R. G. Shao, "Involvement of PI3K/AKT/GSK3beta pathway in tetrandrine-induced G1 arrest and apoptosis," Cancer Biology and Therapy, vol. 7, no. 7, pp. 1073-1078, 2008.

[28] C. Liu, K. Gong, X. Mao, and W. Li, “Tetrandrine induces apoptosis by activating reactive oxygen species and repressing Akt activity in human hepatocellular carcinoma," International Journal of Cancer, vol. 129, no. 6, pp. 1519-1531, 2011.

[29] S. H. Oh and B. H. Lee, "Induction of apoptosis in human hepatoblastoma cells by tetrandrine via caspase-dependent Bid cleavage and cytochrome c release," Biochemical Pharmacology, vol. 66, no. 5, pp. 725-731, 2003.

[30] M. Stücker, C. Springer, V. Paech, N. Hermes, M. Hoffmann, and P. Altmeyer, "Increased laser Doppler flow in skin tumors corresponds to elevated vessel density and reactive hyperemia," Skin Research and Technology, vol. 12, no. 1, pp. 1-6, 2006.
[31] G. L. Semenza, "HIF-1: mediator of physiological and pathophysiological responses to hypoxia," Journal of Applied Physiology, vol. 88, no. 4, pp. 1474-1480, 2000.

[32] S. A. Siefert and R. Sarkar, "Matrix metalloproteinases in vascular physiology and disease," Vascular, vol. 20, no. 4, pp. 210-216, 2012.

[33] G. J. Mizejewski, "Role of integrins in cancer: survey of expression patterns," Proceedings of the Society for Experimental Biology and Medicine, vol. 222, no. 2, pp. 124-138, 1999.

[34] J. D. Hood and D. A. Cheresh, "Role of integrins in cell invasion and migration," Nature Reviews Cancer, vol. 2, no. 2, pp. 91-100, 2002.

[35] A. Bianchi-Smiraglia, S. Paesante, and A. V. Bakin, "Integrin $\beta 5$ contributes to the tumorigenic potential of breast cancer cells through the Src-FAK and MEK-ERK signaling pathways," Oncogene, 2012.

[36] Y. H. Kang, N. Y. Ji, S. R. Han et al., "ESM-1 regulates cell growth and metastatic process through activation of NF- $\kappa \mathrm{B}$ in colorectal cancer," Cellular Signalling, vol. 24, no. 10, pp. 19401949, 2012.

[37] Y. H. Kang, N. Y. Ji, C. I. Lee et al., "ESM-1 silencing decreased cell survival, migration, and invasion and modulated cell cycle progression in hepatocellular carcinoma," Amino Acids, vol. 40, no. 3, pp. 1003-1013, 2011.

[38] F. Guo, Z. Zhou, Y. Dou, J. Tang, C. Gao, and J. Huan, “GEFH1/RhoA signalling pathway mediates lipopolysaccharideinduced intercellular adhesion molecular-1 expression in endothelial cells via activation of p38 and NF- $\kappa \mathrm{B}$," Cytokine, vol. 57, no. 3, pp. 417-428, 2012.

[39] P. Melotti, E. Nicolis, A. Tamanini, R. Rolfini, A. Pavirani, and G. Cabrini, "Activation of NF- $\kappa$ B mediates ICAM-1 induction in respiratory cells exposed to an adenovirus-derived vector," Gene Therapy, vol. 8, no. 18, pp. 1436-1442, 2001.

[40] S. Y. Tung, S. F. Chang, M. H. Chou et al., "CXC chemokine ligand $12 /$ stromal cell-derived factor-1 regulates cell adhesion in human colon cancer cells by induction of intercellular adhesion molecule-1," Journal of Biomedical Science, vol. 19, article 91, 2012.

[41] Y. Usami, K. Ishida, S. Sato et al., "Intercellular adhesion molecule-1 (ICAM-1) expression correlates with oral cancer progression and induces macrophage/cancer cell adhesion," International Journal of Cancer, 2013. 


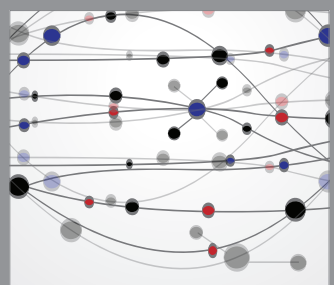

The Scientific World Journal
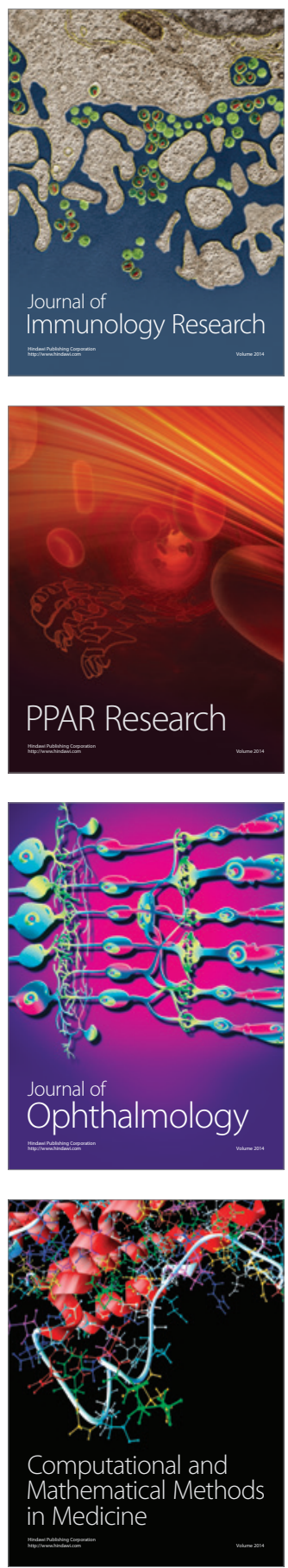

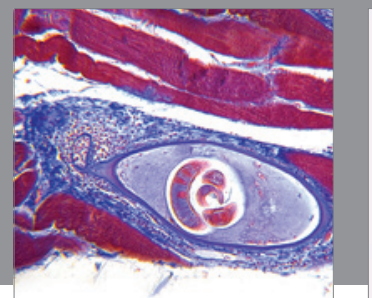

Gastroenterology

Research and Practice
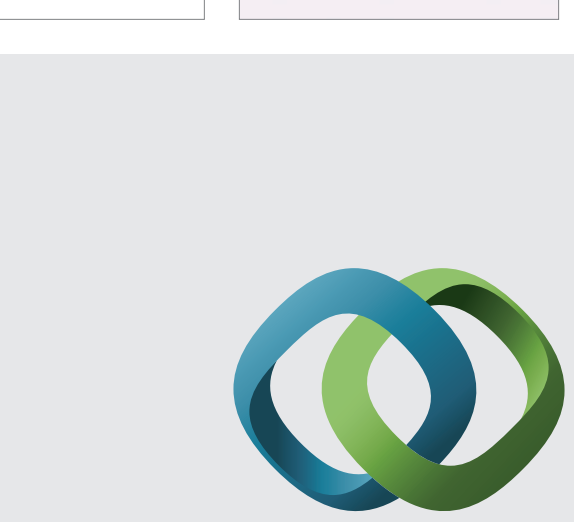

\section{Hindawi}

Submit your manuscripts at

http://www.hindawi.com
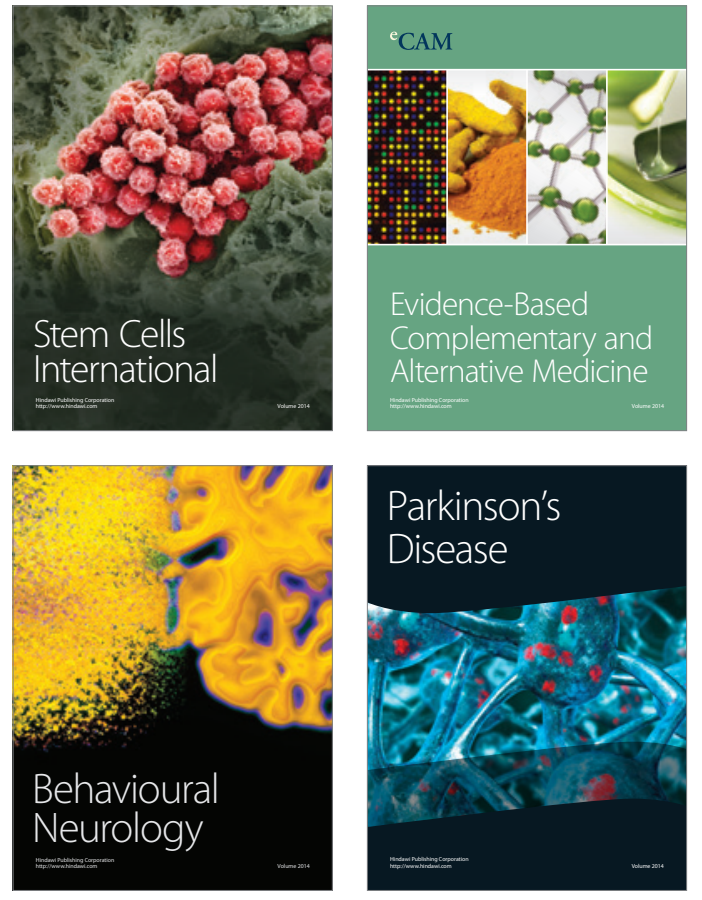
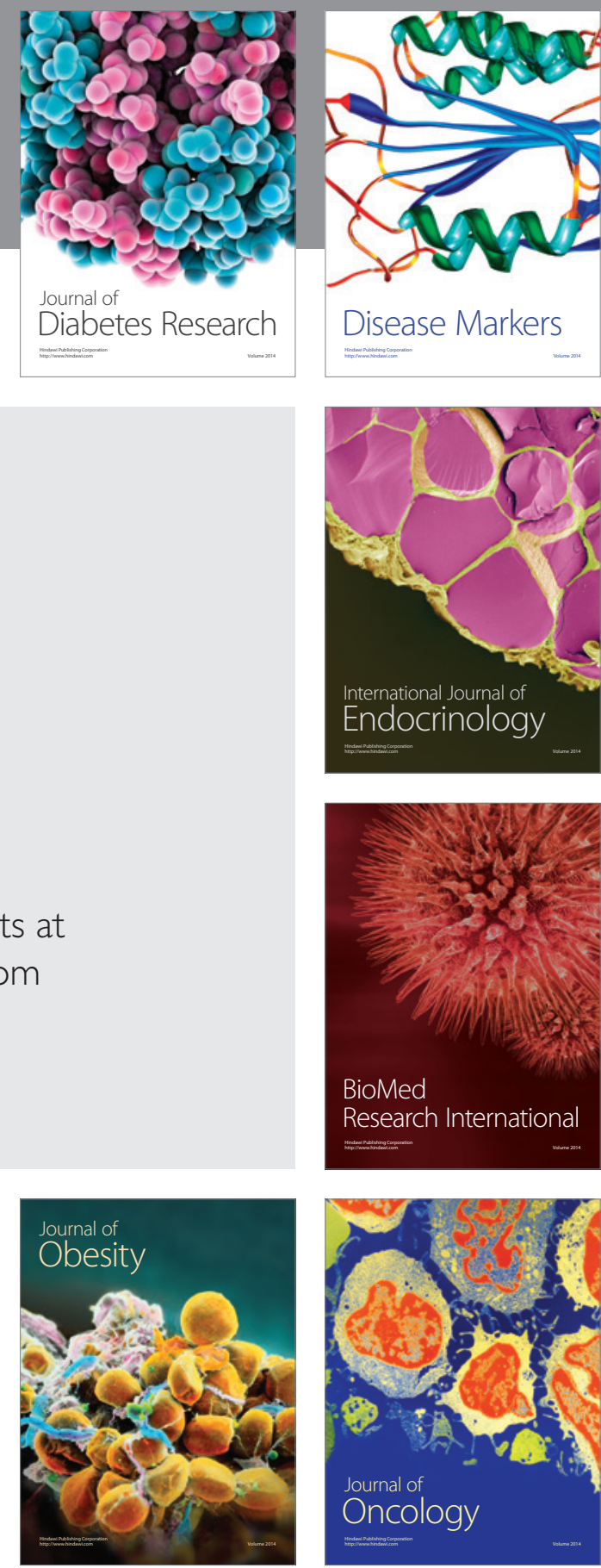

Disease Markers
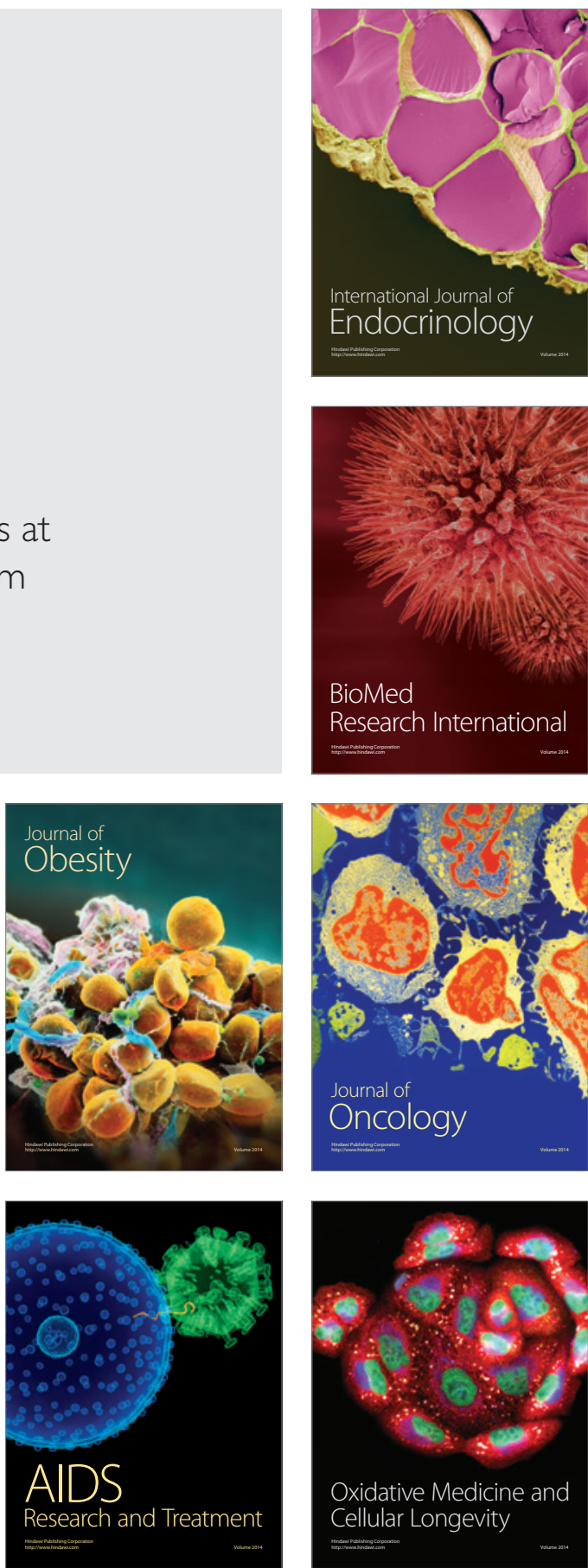